\title{
DE91 004344
}

DOE Technical Report No. DOE/PC/88921-4

Fourth Quarterly (First Annual) Report on Research Grant No. DE-FG22-88PC88921

Title: SPIN-MAPPING OF COAL STRUCTURES WITH ESE AND ENDOR

Principal Investigators: R. L. Belford and R. B. Clarkson

Institution: University of Illinois at Urbana-Champaign

Date: September 1, 1989

US/DOE Patent Clearance is nol required prior to publication of this document.

\section{OBJECTIVES}

The broad goals of this project are to determine by nondestructive means - magnetic resonance techniques - aspects of chemical and physical structures of organic parts of native and treated coals. We also hope to use related methods to follow the course of certain coal cleaning processes with microscopic spatial resolution. More specific project goals include:

1. The nondestructive determination of atomic and molecular structure of sulfur-containing organic species in coal both in its natural state and at various stages during desulfurization;

2. Determination of interatomic distances, numbers, and orientations in individual macerals with differing sulfur content by pulsed EPR microscopy;

3. Development of nondestructive high-resolution microscopic images of internal structure in coal, including chemical information on the location and distribution of sulfur-containing compounds;

4. Determination of sulfur compound chemical structure from highly localized regions in a whole coal sample:

5. By means of the techniques used to accomplish the above goals, to measure the effects of various coal cleaning methods on the molecular forms and spatial distribution of organic sulfur, and on internal structural characteristics like pore size and maceral density;

6. Following by these microscopic methods the rate and extent of solvent intrusion into the pores and mairix of whole coals and separated macerals

These goals describe the scope of the entire three-year DOE project, support for which is enhanced during the first two years by auxiliary support for the Center for Research on Sulfur in Coal, an agency of the State of Illinois. After one year, work is proceeding vigorously in most of these areas.

The work carried out this year mainly addressed goals $1,2,3$, and 6. Very High Frequency EPR, done at $W$-band $(96 \mathrm{GHz}$ ), was for the first time peiformed on coals and separated macerals, addressing Goal \#1. The W-band spectrometer was designed and built in our laboratory, and represents the only instrument of its kind in the world currently studying coal. S-band ( 3 Ghz) pulsed EPR and Electron Spin Echo (ESE) spectroscopiess were employed to develop data on atomic structural parameters of coal (Goal \#2). Again, the instruments used in this work were designed and buill in our laboratory, and are the only devices of their kind used for coal research in the world. The world's first high resolution Magnetic Resonance Imaging was performed on coal samples, and very preliminary data demonstrates that the technique works extremely well (Goals \#3 and \#6). In addition to these main project areas, a very significant amount of experimental and theoretical work was accomplished using coal model compounds. This work is extremely important for the development of new techniques, as well as for the interpretation of data obtained from coals and separated macerals. 


\section{INTRODUCTION AND BACKGROUND}

A major objective in coal science continues to be the nondestructive determination of atomic and molecular structure in the individual cornponents that comprise this heterogeneous mineral. Work on questions seemingly as different as the geologic origin of different macer als aini the effects of various coal cleaning technologies would benefit greatly from detailed atomic information about coal structure. Because of its helerogeneous and complex nature, the microscopic structure of coal will not be entirely understood through the use of a single physical method, and it is not productive to view research in this area as a contest between various techniques. Rather, several different approaches should be used in a complementary way, each contributing part of the information that is needed to develop a more comprehensive and useful map of coal atomic and molecular struclure.

Since nondestructive techniques are all relatively new, most of what we know about the composition of coal cories from destructive methods. Attempts to deduce the native structure of coal from mass spectral, NMR, separation science, and intr ared data on components derived from chemically or thermally treated samples have led to the development of two main approaches for the modeling of maceral structure .. parameters and average molecular construction. Parameters include such average structural information as aliphatic/aromatic carbon ratios and number of aromatic rings per molecule. Average molecular construction approaches attempt to devise a solid maceral structure that will account for the molecular structures found in products. Several examples of this second method are given in the following references $[1,2,3,4,5]$. Figure 1 illustrates one of these structures, that developed by Shinn for an average bituminous coal maceral [3].

The importance of coal models (and detailed information on microscopic struclure) is growing as work on more complex processing methods seeks to relate coal properties with coal molecular and atomic structure. Because of the many uncertainties in

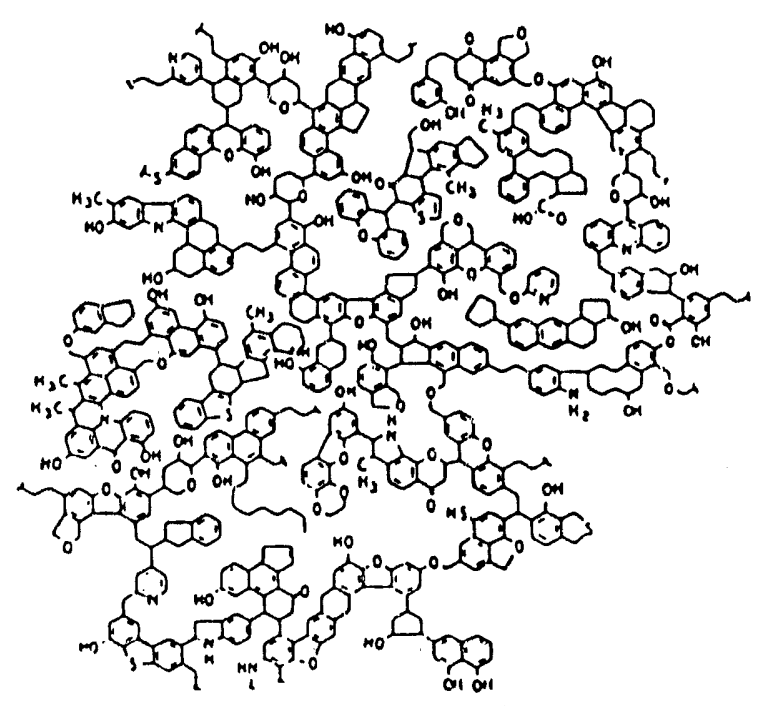

Figure 1. Shinn's model of bituminous coal structure [3].

relating composition data obtained from destructive analytical methods to the native structures in coal, there is a need both for nondestructive approaches to the determination of atomic and molecular structure and for information about specific coal samples rather than statistical information on average properties. Among the relatively few nondestructive methods capable of observing coal structure on 
this microscopic scale, magnetic resonance methods (EPR, NMR, ENDOR, ESE) are showing great promise. This is because these methods have, in many cases, developed theoretical and experimental ways to achieve the 1) sensitivity, and,2) resolution needed to determine microscopic struclure in noncrystalline, opaque, and relatively non-volatile solids like coal.

Among magnetic resonance spectroscopies, none is more sensitive than Electron Paramagnelic Resonance (EPR). It was natural that two of the first studies ever undertaken vith the then-new technique (ca. 1953) were investigations of the unpaired electrons naturally occurring in coal $[6,7]$. From the very beginning of EPR work on coal, it was apparent that the lechnique had enormous sensitivity, but that by itself it lacked sufficient resolution to observe the very small hyperfine splittings that contain information on the atomic and molecular struclure "observed" by the electron. Although NMR usually has the ability to resolve much smaller splittings, it also was unable to achieve the necessary resolution to determine coal molecular struclure directly, and was hampered by sensitivity problems as well. One potential solution to these difficulties emerged with the advent of Electron-Nuclear Double Resonance (ENDOR) spectroscopy, a hybrid technique that can be characterized as NMR with detection by EPR (e.g. NMR resolution with EPR sensitivity), and which is specifically designed to resolve hyperfine interactions.

In 1981, Retcolsky and co-workers reported the first successful resolution of electron-nuclear hyperfine interactions (other than matrix effects) in several Pennsylvania whole coals using ENDOR [8]. They saw hyperfine couplings characteristic of those expected in aromatic hydrocarbons, as well as one coupling possibly from an aliphatic moiety. The similarity of couplings from several coals generally supported the view that macerals may be composed of a limited number of functional groups, linked together in varying combinations and concentrations. Such information is exactly the sort needed to develop specific information on the molecular structure of coal, and demonstrated the potential of the technique. The overall approach of using unpaired electrons in coal to observe atomic and molecular structure was termed HYPERFINE FINGERPRINT SPECTROSCOPY.

For the past five years, our laboratory has worked un extensions of the ENDOR technique as applied to the structural determination of coal. We have developed computer-controlled instrumentation capable of performing complex ENDOR experiments involving two variables, which we term 2 dimensional ENDOR, and which have shown tremendous promise in increasing still further the spectrai. resolution of the technique for coal work. We also have developed theoretical methods for analyzing the powder ENDOR spectra oblained from coal samples, and have performed many experiments on model systems as well. We are developing a library of ENDOR spectra from powders containing modei compounds (eg, anthracene, naphthalene, pyrene, perylene, dibenzothiophene, etc), which we will use to begin the analysis of data from whole coals [9]. In addition, we have buill a time-domain (pulsed) EPR spectrometer optimized for coal studies, and have begun to employ it to do Electron Spin Echo (ESE) spectroscopy with excellent results [10].

The importance in coal research of nondestructive analytical methods cannot be overemphasized. Not only do these methods yield information about truly unmoditied coal, they allow accurate comparisons to be made between raw coal and material that has been treated by various cleaning technologies, thus providing benchmark data on the effects of cleaning as well as on the native coal. Some questions cannol be answered by any other approach -- questions concerning the distribution of organic sulfur compounds along micropores in coal being a case in point.

Among nondestructive methods, magnetic resonance techniques represent some of the very few capable of getting information about both chemical composition and compound location. We believe that the electron magnetic resonance approaches developed by us over the last five years (with partial 
support from both the $U \subset \subset$ Department of Energy and the State of Illinois, through the Center for Research on Sulfur in Coal) offer a unique analytical view of organic sulfur in coal. Specifically, and with reference to the six goals given above, we believe that this program is making significant contributions to our understanding of organic parts of coal, including organic sulfur, in the following ways:

1. Chemical characterization: nondestructive determination of the molecular structures of sulfurcontaining organic compounds in both whole coal and in separated macerals. This is a goal of enormous importance. Applications of such information to the analysis of coal, as well as to the design, optimization. and evaluation of coal cleaning technologies are too numerous to list. By obtaining hyperfine fingerprint spectra from whole coal and separated macerais, our electron magnetic resonance technique can measure components of this structure, and by the sulfur $g$ shifts, fingerprints associated with sulfur compounds can be identified.

2. Physical characterization: obtaining precise data $( \pm 0.1 \mathrm{~A})$ on the interatomic distances, number density, and arrangement of atoms in individual macerals. This, to be accomplished by high. resolution pulsed EPR microscopy, will help us to understand the influence of sulfur on the physical characteristics of coal, processed coal, and separated macerals. The advent of excellent methods of maceral separation (eg density gradient centrifugation) has focussed attention on the large differences in organic sulfur content of various maceral components. Details of the physical differences between different maceral types will improve understanding of these sulfur concentration differences, and will help to suggest optimal strategies for sulfur removal. The information also will be important in assessing the effects of various coal cleaning technologies. Our electron magnetic resonance method can nondestructively provide this information with a precision unriva!ed by other techniques.

3. Physical characterization: micron-resolution spatial imaging of coal samples. In recent years, magnetic resonance imaging has made dramatic advances in image clarity, resolution, and chemical information content. Our laboratory has developed unique instrumentation to do electron magnetic resonance imaging microscopy, which we propose to use to study the distribution of sulfur-containing species and maceral domains in whole coal and separated materials. Obvious areas of importance to coal science include: 1) the nondestructive mapping of sulfur compounds in different macerals and at various boundaries such as pores and inorganic mineral inclusions in whole coal, 2) analysis in space and time of solvent penetration into the coal matrix. Modern digital image reconstruction techniques allow us to develop images (maps) of only sulfur compounds, or of other, more general organic chemical species with a resclution of 1 micron (or better).

4. Physical and chemical characterization: obtaining hypertine fingerprint data from selected volume elements in a piece of whole coal. This is another important and complementary capability of imaging technology. Our goal here is to gel chemical structure information on sulfur compounds in individual macerals without the necessity of physically separating the components. Such a nondestructive "magnetic resonance dissection" is possible because of the tremendous sensitivity of our electron magnetic resonance spectroscopy. Such a capability would be very useful in the routine analysis of coal, as well as in evaluating the effects of coal cleaning on different portions of the coal, without actually destroying the samples and introducing uncertainties into results.

We have made substantial progress this year implementing and using these new experimental methods to non-destructively characterize high-sulfur coals. 


\section{EXPERIMENTAL PROCEDURES}

Because this program is based on several different magnetic resonance techniques, a detailed description of each method will nol be given here. Instead, the basic physical principles underlying the procedures will be described in enough detail to explain the experiments, results, and conclusions that follow in subsequent sections.

\section{1) VHF W-Band EPR}

The fundamental motivation for building a spectrometer to perform the EPR experiment at a magnetic field of $3.4 \mathrm{~T}$. instead of at the usual $0.34 \mathrm{~T}$. is to improve spectral resolution and sensitivity of the technique to sulfur-containing compounds. The relationship between magnetic field strength and sensitivity to sulfur can be better undersiood by considering the energy of an unpaired electron in an external field, B. If the atomic or molecular orbital of the electron is nondegenerate $(L=0)$, then the "spin only" energy is given by:

$$
H_{\mathrm{ez}}=\mathrm{B}_{\mathrm{e}} \times \mathbf{B} \cdot \mathbf{g} \cdot \mathrm{S}
$$

where $H_{e z}$ is the spin Hamiltonian (electronic Zeeman interaction only), $B_{\mathrm{e}}$ is the Bohr magneton, $B$ is the external magnetic field, $g$ is the $g$-tensor, and $S$ is the electron spin operator. In this case, when the electron possesses only spin angular momentum, the g-tensor is isotropic and has the free electron value $g_{\mathrm{c}}=2.00232$.

When the electron is in an orbital with angular momentum $(L>0)$, either by itself or via coupling to excited states, then the orbital angular momentum mixes with the spin angular momentum. Our Zeeman spin Hamiltonian now contains another term, reflecting this additional interaction:

$$
\begin{aligned}
H_{02}= & B_{0} B \cdot L+g_{c} B_{0} B \cdot S, \text { or } \\
& =B_{0} B \cdot\left(L+g_{c} S\right)
\end{aligned}
$$

In addition to modifying the electronic Zeeman energy, the orbital angular momentum of the unpaired electron (or the magnetic dipole moment induced by the orbital motion) also interacts directly with the unpaired electron, crealing an additional energy term:

$$
H_{\mathrm{so}}=\lambda L \cdot S
$$

where $\lambda$ is the spin-orbit coupling constant, a term proportional to the nuclear charge of the atom $(Z)$ and $1 / r_{3}$, where $r$ is the orbital radius. $\lambda$ may be positive or negative, reflecting the fact that the local orbital field may add to or subtract from the external static field.

The total spin energy for this system now is written:

$$
H_{\text {spin }}=H_{\mathrm{e} z}+H_{\mathrm{so}}=B_{\mathrm{e}} B \cdot\left(L+g_{\mathrm{c}} \mathrm{S}\right)+\lambda L \cdot S \text {. }
$$

The effect of orbital angular momentum is to alter the energy of the spin in an external magnetic field. We now must remember that in the EPR experiment, we measure the energy represented by $\mathrm{H}_{\text {spin, }}$, not by scanning frequericy, but by scanning magnetic field. Field positions of resonance lines are related to energies by the simple resonance equation: 


$$
h_{v}=g_{\text {eff }} \beta_{0} B_{0} \text {. }
$$

where all symbols have their customary meaning, and $g_{\text {eff }}$ is an "effective g-value" that characlerizes the position of the line (analogous to the chemical shift parameter in NMR). If $L=0$, then $g_{\text {eff }}=g_{0}$. as can be seen in Equ. (iv); if $L>0$, then $g_{e f f}$ will deviate from $g_{\mathrm{e}}$ in a way determined by $L$ and $\lambda$. In the coal system, spin-orbil coupling $\left(\mathbf{H}_{\mathrm{so}}\right)$ is primarily responsible for this deviation, and this key fact allows us to observe the presence of organic sulfur compounds in the EPR spectra.

\section{2) Pulsed S-band EPR and ESE}

Although the ideas behind $E S E$ techniques are as old as magnetic resonance itself (1946), the general application of spin echo methods to paramagnetic systems has occurred in only the last few years with the advent of very high speed digital electronics under computer control. Unlike the familiar continuous wave (cW) EPR experiment, which has been available in commercial instrumentation for nearly 20 years, ESE spectroscopy is not yet a commercially available technique, and only a dozen or so laboratories throughout the world currently perform the experiment. For an excellent review of the theory and early applications of ESE spectroscopy, the book by Kevan and Schwartz is recommended [11].

Unlike cW EPR, ESE is a pulsed microwave experiment. Very short microwave pulses excite the paramagnetic spin system, and the time evolution of the magnetization is monitored. Certain pulse schemes, characterized by the number of degrees the magnetization vector is tipped by each pulse, are known to generate a strong instantaneous magnelization in the sample following the sequence. Typical pulse schemes known to induce this rephasing or "echo" effect are $90^{\circ}$-T-180 (Hahn echo sequence) and the $90^{\circ}-1-90^{\circ}-\mathrm{T}-90^{\circ}$ (stimulated echo sequence). We have found the stimulated echo sequence, diagrammed in Figure 2, to be most useful in our coal studies.

Figure 2. Timing diagram of stimulated-echo pulse sequence. Typical pulse 40nsec; times 1 and $T$ range from .001 to $1 \mathrm{msec}$

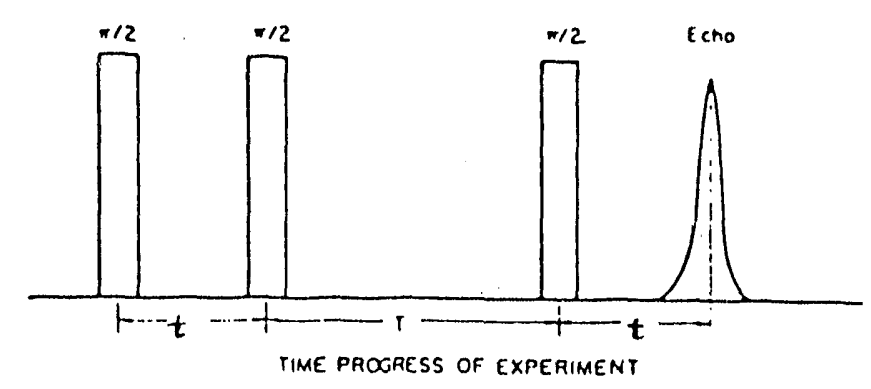

ESE spectroscopy has many advantages over the more conventional cW EPR. Chiel among the advantages for our coal work is that, like ENDOR, the technique may be used to obtain spectra which resolve hyperfine structure that is obscured in $\mathrm{cw}$ spectra by inhomogeneous line broadening. In order to understand how ESE can be made to accomplish this task, let us look at the characteristics of the information obtained in the experiment. 
In a typical stimulated echo experiment done on coal, the time interval $t$ between the first and second $90^{\circ}$ pulse is set at a fixed value during the experiment, while the interval $\mathrm{T}$ between the second and third pulses is incremented in steps, as shown in Figure 3. The amplitude of the echo induced by the sequence is measured as a function of the delay time $T$ between the second and third pulses. In the absence of any interactions between the unpaired electrons being observed and neighboring nuclei with non-zero magnetic moments, the variation of the echo amplitude $V$ with the delay time $T$ is given by the simple exponential function:

$$
V_{\text {echo }}(21+T)=V_{0} \exp \left[(21+T) T_{11}\right]
$$

where $\mathrm{Tm}$ is the phase memory time of the individual spin packets. The exponential decay curve describing the echo amplitude as a function of $\mathrm{T}$ is called the electron spin echo envelope.

Figure 3. Measurement by the method of stimulated echoes, showing ESEEM.

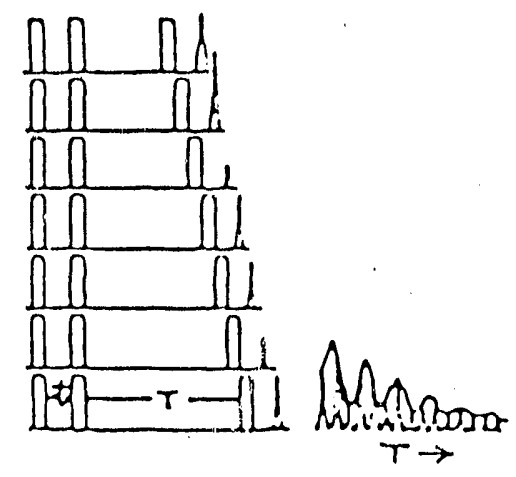

If our unpaired spins experience hyperfine interactions with neighboring nuclei, these interactions will manifest themselves as modulation patterns in the ESE envelope. This phenomenon is thus known as electron spin echo envelope modulation (ESEEM). For an $S=1 / 2,1=1 / 2$ system with isolropic $g$ values (a very good approximation in the case of coal) and two hyperfine interactions characterized by frequencies $f_{a}$ and $f_{b}$, the ESEEM paltern (without echo decay) is given by:

$V_{\bmod }(2 t+T)=1-k\left\{\sin ^{2}\left(f_{d} t / 2\right) \sin ^{2}\left[f_{t}(t+T) / 2\right]+\sin ^{2}\left(f_{b} t / 2\right) \sin ^{2}\left[f_{d}(t+T) / 2\right]\right\}(v i)$

In this expression, $k$ is the so-called modulation depth parameler, propiortional to $B_{0}{ }^{2}$ in the limit of small hyperfine interactions $\left(B_{0}\right.$ being the value of the static external magnetic field). For more than one nucleus, $V_{\text {mod }}$ is the product of the modulation functions of the individual nuclei:

$$
v_{\bmod }=V_{\bmod }\left(I_{1}\right) v_{\bmod }\left(I_{2}\right) \cdot V_{\bmod }\left(I_{3}\right) \ldots
$$

Thus, for the case of $n$ like nuclei coupled identically to an electron spin, the overall modulation is given by the modulation function for one nucleus raised to the $n$th power. The overall modulated signal in the time-domain spectrum is then the product of these two functions:

$$
V_{\text {echo }}(2 t+T)=V_{\text {decay }} V_{\text {mod }}
$$


A Fourier transform of the ESEEM pattern results in a frequency domain spectrum exhibiting linewidths that are characteristic of individual spin packets rather than of the entire envelope of spin packets comprising the inhomogeneously broadened lines observed by cW EPR. Since the modulation frequencies can be analyzed by an ENDOR-like theory, this technique provides us with a powerful alternative way of resolving the hyperfine interaction energies normally obscured in the cw spectra of whole coal. This information, in turn, allows us to sludy the environment experienced by radicals in coal, as well as giving us information on the structure of the radicals themselves. ESE and ENDOR thus provide two complementary routes to the study of coal molecular and atomic structure.

Finally, we can simulate the time-domain ESEEM pattern described in Equ. (vii) on the basis of coal structural models involving distances between the unpaired electron and nuclei with non-zero magnetic moments, numbers of atoms, and orientations. By fitting theoretical simulations to experimental data, detailed atomic structural information is obtained.

\section{3) Magnetic Resonance Imaging}

Three dimensional EPR imaging has been developed in the Illinois EPR Research Center to achieve precise nondestructive microscopic spatial resolution of internal structures in opaque objects. Good reviews of the method can be found in References 12, 13, and 14. The symmetry constraints placed on samples by two dimensional methods are completely removed in this implementation.

Spatially resolved data are obtained with a sel of rectangular transverse field gradient coils, mounted on circular $z$-gradient coils, and rotated about the $z$ axis to produce any desired gradient. The field gradients act as scales to measure distances or positions, and the raw data comes encoded in terms of magnetic fields. Through sophisticated computer back reconstruction algorithms, this fieldencoded data is used to construct three dimensional pictures of the object, including high-resolution microscopic images of internal structures. The software controlling data acquisition currently can obtain images of a small object in a series of 200 micron "slices" of arbitrary orientation (the orientation can be decided on after the experiment is finished and the data is being processed).

It also is possible to image protons by NMR imaging techniques. Thus far we have pertormed preliminary experiments on proton imaging of solvents diffused into coal. These early results, which we shall report in more detail in the first quarterly report of the second year, seem extremely promising for studying solvent penetration in coal.

\section{RESULTS AND DISCUSSION}

1) VHF W-band EPR

The coal used in this study was an llinois \#6 (Herrin), provided by the lllinois State Geological Survey. Chemical analysis gave (wt \%, dry and ash free): $H(5.25), C(81.15), N(1.78), O$ (8.65), S (3.17). Analysis of sulfur by type gave ( $w t \%$, dry and ash free): sulfate sulfur (0.115), pyritic sulfur (2.32), organic sulfur (0.74). The maceral composition of the coal was $87 \%$ vitrinite, $4.4 \%$ liptinite, and $8.6 \%$ inertinite. A sample of vitrinite concentrated from this coal also was sludied. The chernical analysis of the vitrinite gave ( $W . \%$, dry and ash free): $H(5.10), C(79.77), N(1.80), O(12.49), S(0.74)$. Analysis of sulfur by type gave: pyritic sulfur $(0.06)$, organic sulfur $(0.68)$.

Coal samples were powdered to 100 mesh and some were evacuated to a pressure $<10^{-6}$ Torr 
for at least 24 hours. These were sealed in quartz glass tubes for examination by EPR. Other samples were left open to air. Little or no dependence on evacuation was noted in the EPR spectra of whole coal and vitrinite samples. Fusinites separated from this coal showed a very large linewidth variation with evacuation.

\section{W-band EPR spectrometer}

The W-band spectrometer was designed to operate at a nominal frequency of $96 \mathrm{GHz}$. Either a digitally stabilized Gunn oscillator with a power output of $35 \mathrm{~mW}$ or a klystron with a power rating of $400 \mathrm{~mW}$ can be used. In the experiments described here, the gunn oscillator was used, in conjunction with either a low-Q terminated waveguide resonator or a higher-Q cavity resonator operating in the cyllindrical $T E_{012}$ or $T E_{013}$ mode. Microwave power at the cavity was measured to be between 8 and $11 \mathrm{~mW}$. Frequency was monitored and locked with an EIP digital counter. The actual operating frequency in these experiments was about $94.2 \mathrm{GHz}$. The main magnetic field $\left(B_{0}\right)$ was provided by a superconducting magnet that originally had been part of a Vai ian XL-200 NMR spectrometer system, and that was donated for this research by Varian. Associates, Inc. The superconducting field was se! at the $\mathrm{g}=2$ value of $3.36 \mathrm{~T}$. The magnet room temperature bure was outfitted with sweep coils that provided digitally controlled field sweeps of up to 280 Gauss. Fields were measured with an autotracking NMR teslameter (Metrolab).

Sinusoidal field modulation, 20 or $100 \mathrm{kHz}$, was used, and the signal was ultimately lock-inamplified and stored in an IBM PC computer for analysis. The powdered materials in $0.8-\mathrm{mm}$ id quartz capillaries were loaded into the cylindrical cavity. Fig. 4 shows a block diagram of the instrument.

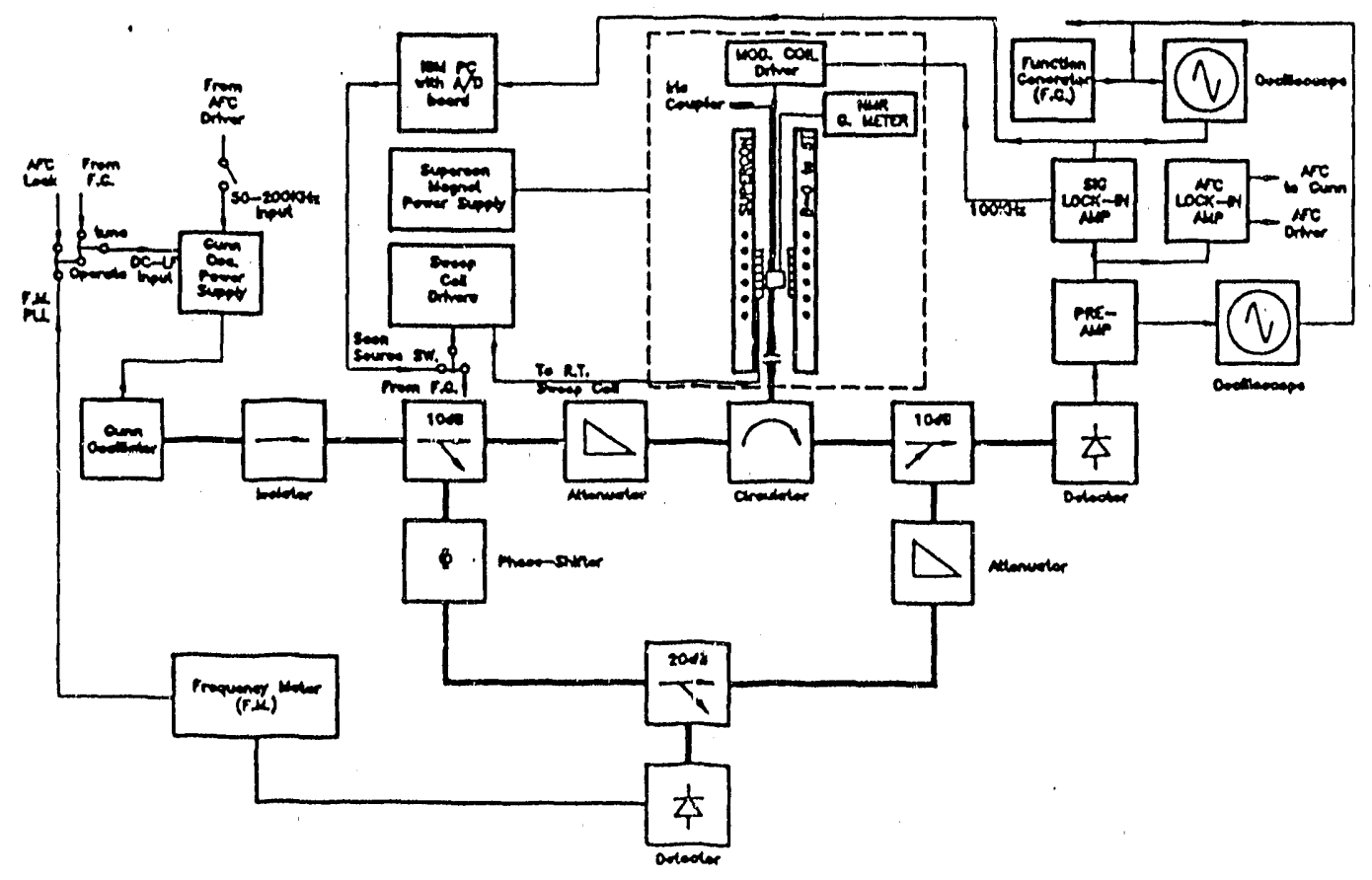

Figure 4. Block diagram of the W-band spectrometer. 
Recently, Freed listed three EPR groups with spectrometers operating in the very high frequency region [15]: Lebedev and co-workers (135 GHz), Möbius $(94 \mathrm{GHz})$, and Freed $(250 \mathrm{GHz})$. Earlier success.." millimeter wave EPR spectrometers were built by A. A. Galkin et al. [16] and by R. J. Wagner, et. al. [17]. Weber and co-workers recently have reported successfully operating a $95 \mathrm{GHz}$ pulsed EPR spectrometer [18]. Our W-band spectrometer thus joins about six other reported EPR instruments that have operated above $90 \mathrm{GHz}$, and is the newest entry in this millimeter-wave group. therein).

Spectra' simulations were performed by techniques outlined in Reference [9] (and citations

\section{RESULTS}

Figures 5(a-c) show EPR spectra of vitrinite concentrate obtained from lllinois \#6 coal, measured at $X(9.5 \mathrm{GHz}), Q(35 \mathrm{GHz})$, and $W$-band $(96 \mathrm{GHz})$. Only at $W$ is the low-field shoulder resolved, although it is becoming evident at $Q$-band in the form of increased asymmetry of the single resonance line.
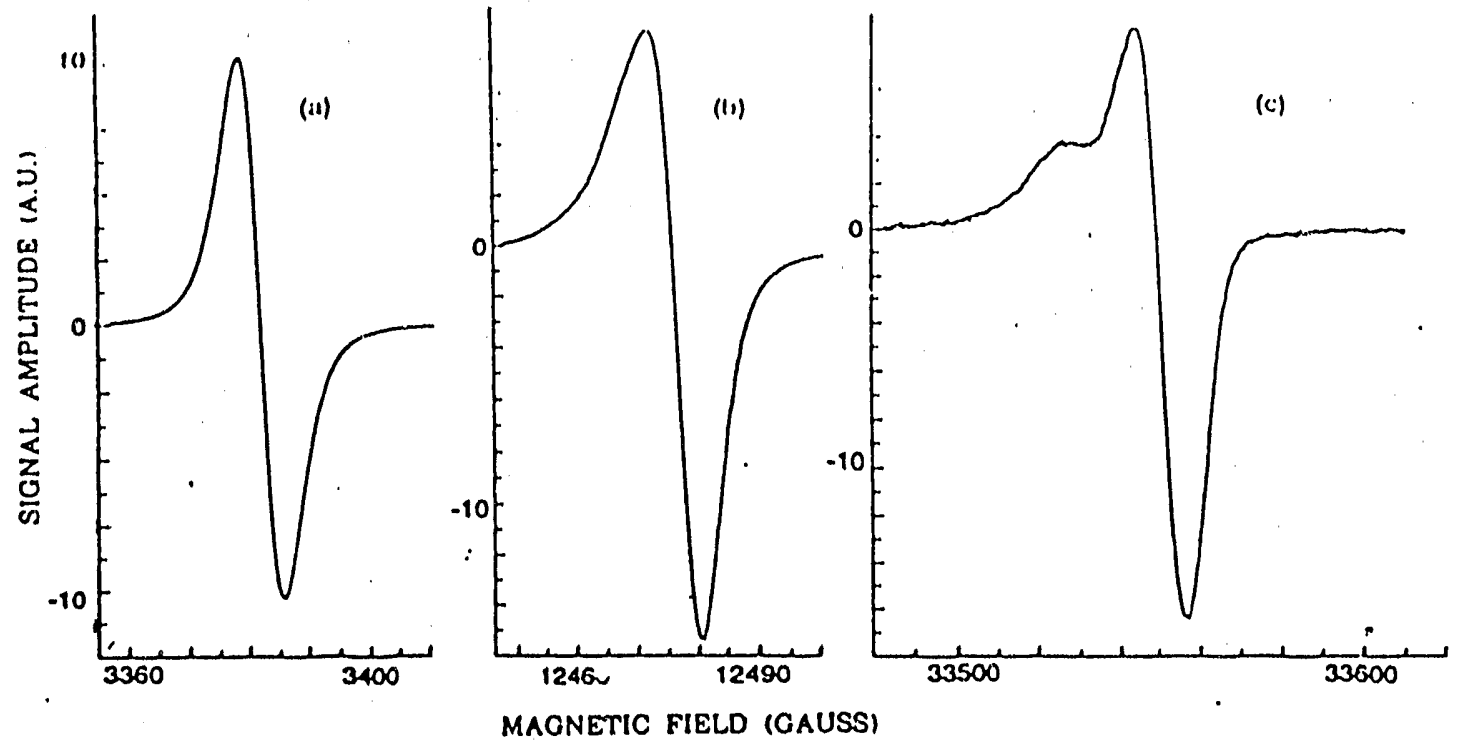

Figure 5. EPR spectra of vitrinite from III. \#6 coal. (a) X- (9.5 GHz), (b) Q- (35 GHz), (c) W-band (94 GHz).

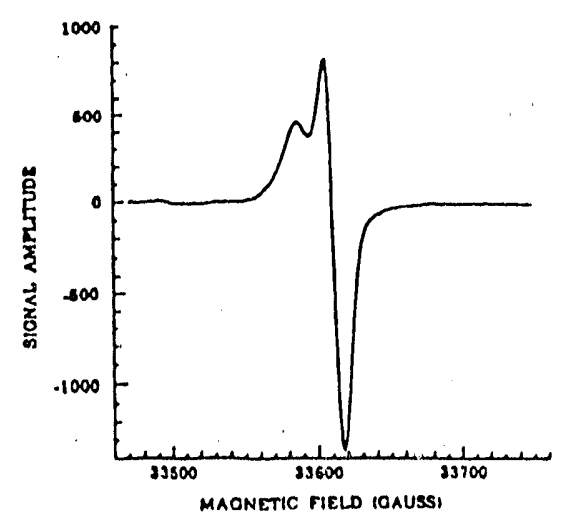

Figure 6. W-band EPR spectrum of the whole Illinois $\# 6$ coal. 
The increase in spectral resolution also is seen in the EPR spectra of the whole coal, a W-band spectrum of which is shown in Figure 6.

A comparison of Figures $5(\mathrm{c})$ and 6 shows differences in the W-band spectra of the whole coal and the vitrinite separated from it. If the amplitude of the vitrinite spectrum is adjusted carefully relative to the whole coal signal, as shown in Figure $7(a)$, then subtracting vitrinite from whole coal leaves the smaller signal shown in Figure 7 (b). The narrow signal resulting trom subtraction is probably due in large part to fusinite in this coal. Double integration of the scaled vitrinite and whole coal spectra results in numerical values for the areas under each spectral line that are proportional to the number of unpaired electron spins contributing to each spectrum. The ratio for this pair of double integral values is: vitrinite/whole coal $=0.84$. This number agrees very well with the maceral analysis that reported this coal to be $87 \%$ vitrinite.
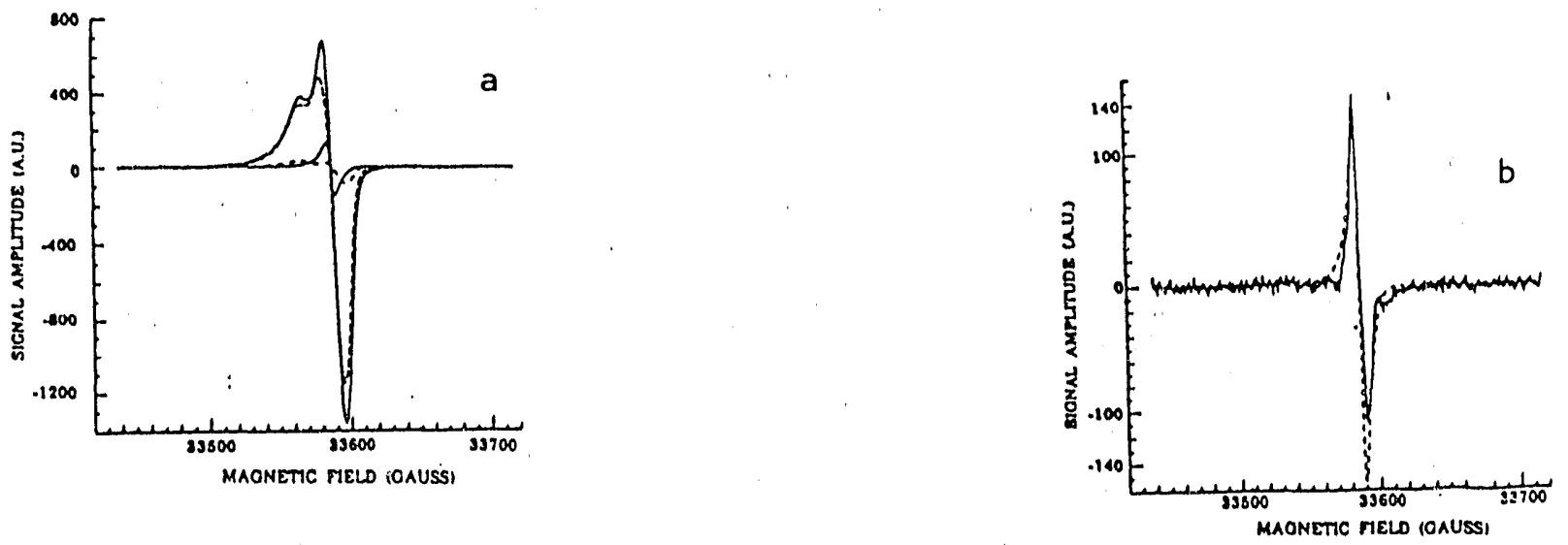

Figure 7. (a) Scaled W-band EPR spectra. ( ) Illinois \#6, (-.-) vitrinite, $(\cdots)$ fusinite, $(\cdots \cdots)$ sporinite. (b) Difference between whole coal and vitrinite separated from it.

\section{DISCUSSION}

For unpaired electrons in many simple conjugated aromatic hydrocarbon radicals, the most important energy term involving electron spin $\mathrm{S}$ is the Zeeman interaction, given by:

$H_{z}=g B B \cdot S$

where $B$ is the externally applied magnetic field, $B$ is the Bohr magneton, and $g$ is the gyromagnetic ratio or g-factor. In such systems, the urifaired electron is associated only with carbon and hydrogen atoms, and the $g$-factor is nearly isotropic and cin be represented by a scalar constant, $g_{0}\left(g_{0}=2.0023\right)$. Very small deviations from $g_{0}$ are the result of the interaction of the electron spin (S) with its own orbital angular momentum (L), as well as from contributions from low-lying excited states. The magnitude of the $L, S$ interaction is sometimes parameterized by a constant, $\lambda$, called the spin-orbit (SO) coupling constant, which is different for each element (and in some treatments, for the same element in different orbital hybrids). The very small deviations in $\mathrm{g}$ resulting from this interaction in conjugated aromatic hydrocarbons are given by:

$g_{x, y, z}=g_{0}+\lambda / K$ 
where $K$ is a constant dependent on the electronic orbitals involved. SO coupling has the effect of making $g$ larger than $g_{0}$, and of introducing anisotropy to the g-factor. Such very small effects usually are not detected by EPR at conventional frequencies, and even at W-band, their contributions usually appear in the form of spectral line broadening.

As the atomic number increases, $\lambda$ increases across the first rows of elements in the periodic table, ranging from $29 \mathrm{~cm}^{-1}$ for carbon to $382 \mathrm{~cm}^{-1}$ for sulfur. We therefore expect to observe the presence of sulfur, and to a lesser extent oxygen, in the W-band EPR spectra of coal primarily as spectral intensity occurring at $\mathrm{g}$ values larger than those of pure hydrocarbons.

Precise effects of heteroatoms on the EPR lineshape of coal will, of course, depend on the manner in which these elements are included in the molecular framework of the organic macerals, as well as the distribution of radicals relative to tha: framework. While it is very encouraging :o note the excellent agreement between the ratio of unpai:ed spins in the separated vitrinite/whole coal $(84 \%)$ and the maceral analysis for vitrinite $(87 \%)$, this result should not be interpreted as meaning that unpaired electrons are uniformly distributed throughout macerals. Much more critical tests of radical distribution will be the ratio of unpaired electrons found in the less abundant macerals/whole coal. The ability of Wband EPR to provide this information on separated macerals, with resolution that permits the deconvolution of whole coal spectra into contributions from individual macerals with good quantitative precision, opens the way for better analysis of maceral composition by this technique.

Direct simulation of the vitrinite EPR lineshape as if it were the spectrum of a single species resulted in $g_{1}=2.0023, g_{2}=2.00274, g_{3}=2.0042$; see Figure 8 . Actually, chemical and spectroscopic evidence suggests that the EPR spectrum of separated vitrinite has contributions from two or more radical species with different heteroatom compositions, so this sirnulation is useful for roughly identifying the g-values of the main features of the spectrum, but should not be viewed as implying that the single species model is correct. The lower two $g$-values $\left(g_{1}\right.$ and $\left.g_{2}\right)$ are, in fact, just those observed for many different conjugated aromatic hydrocarbon radicals, and predicted theoretically by Stone [19], and it is tempting to see the vitrinite spectrum as a composite formed from pure hydrocarbon and heteroatomic radical contributions. In all probability, the low-field peak in this spectrum is associated with, heteroatomic radicals, since SO coupling to sulfur (or oxygen) is the most likely mechanism to account for the higher value of $g_{3}$. The rather poor low-field (high g-value) fit of the single species simulation then could reflect this composite character. Clearly, this issue of spectral and sample heterogeneity is of the utmost importance for the correct interpretation of EPR spectra from fossil fuels, even when well-separated and purified, and we are presently working to shed more light on the problem.

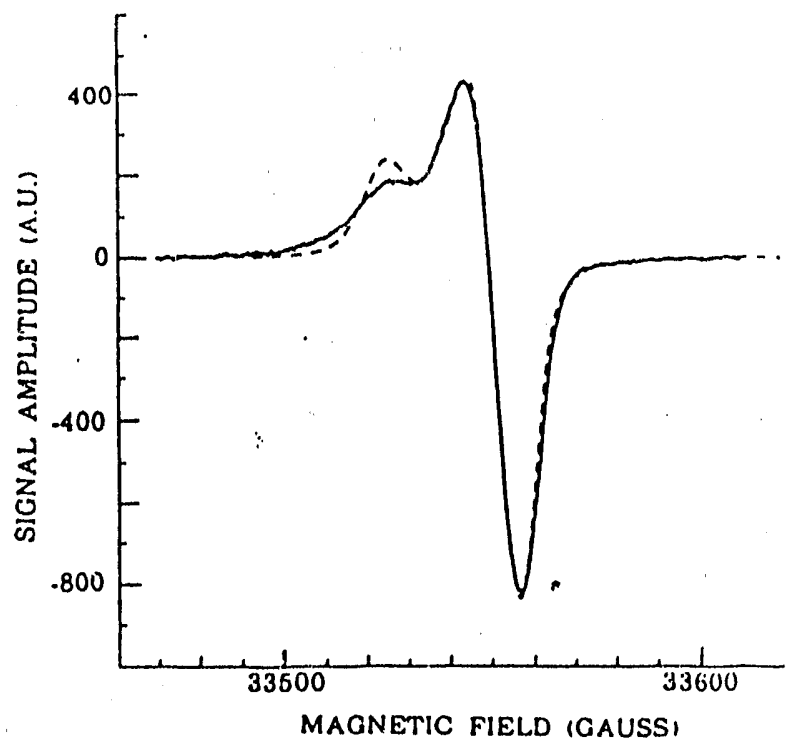

Figure 8. Expt (-) and theor'l (---) W-band spectra of separated vitrinite, assuming a single radical species. 

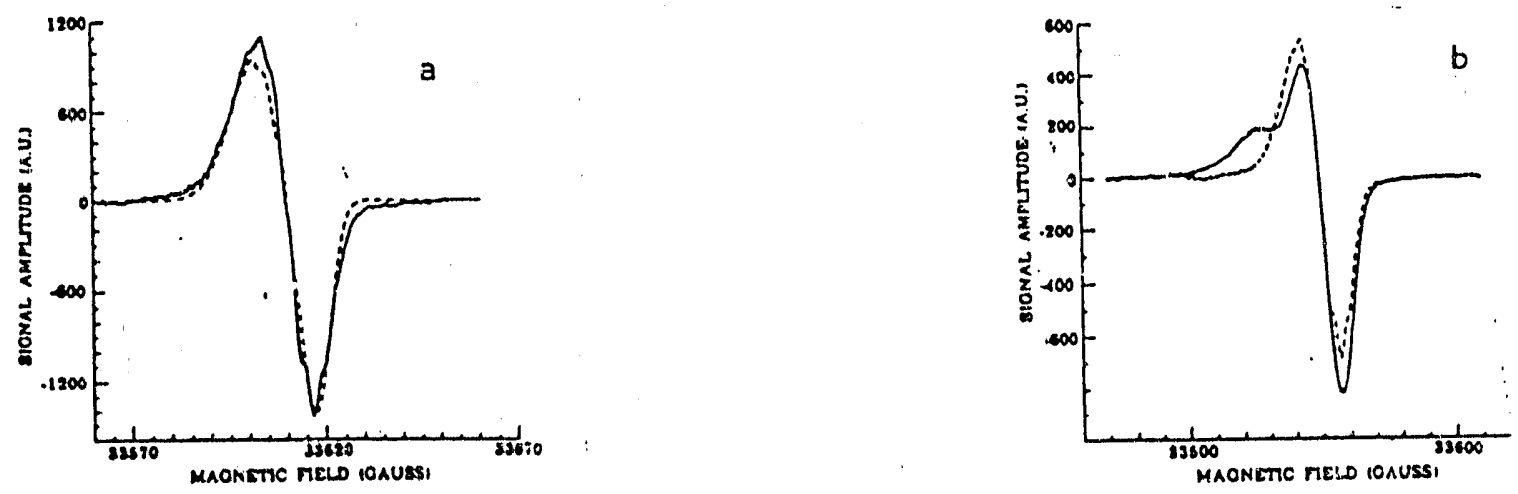

Figure 9. (a) W-band spectrum of perylene $(+)($ vitrinite $(\ldots$, ) and perylene $(+)(-$.$) spectra.$

), theoretical simulation (---). (b) Superposition of

One promising approach is to compare W.band EPR spectra from coal with those of model compounds believed to typ:ty organic structures in coal. Figure 9(a) shows a spectrum of perylene cation radicals, together with a preliminary simulation which used $g_{1}=2.0024, g_{2}=2.0030$, and $g_{3}=$ 2.0032, proton hyperfine values obtained experimentally by ENDOR [20], and spin packet iinewidths determined by electron spin echo measurements. Figure $9(b)$ shows the perylene(t) spectrum superimposed on a vitrinite $W$-band spectrum, to illustrate how a portion of the coal resonance lineshape might originate from conjugated aromatic hydrocarbon radicals containing no heteroatoms.

Figure 10(a) shows a $W$-band spectrum of dibenzothiophene cation radicais with a preliminary simulation $\left(g_{1}=2.004, g_{2}=2.006, g_{3}=2.012\right)$. The large SO coupling with sulfur is seen in the higher $g$-values and much more asymmetric lineshapes. Attar and Dupuis reported that thiophenes are the most abundant form of organic sulfur in Illinois coals (thiophenic, 58\%; Ar-S-Ar, 20\%; R-S-R, 18\%; Ar$\mathrm{SH}, 15 \% ; \mathrm{R}-\mathrm{SH}, 7 \%$ ), so this model compound may well indicate the sulfur-related sffects that are to be expected [21].
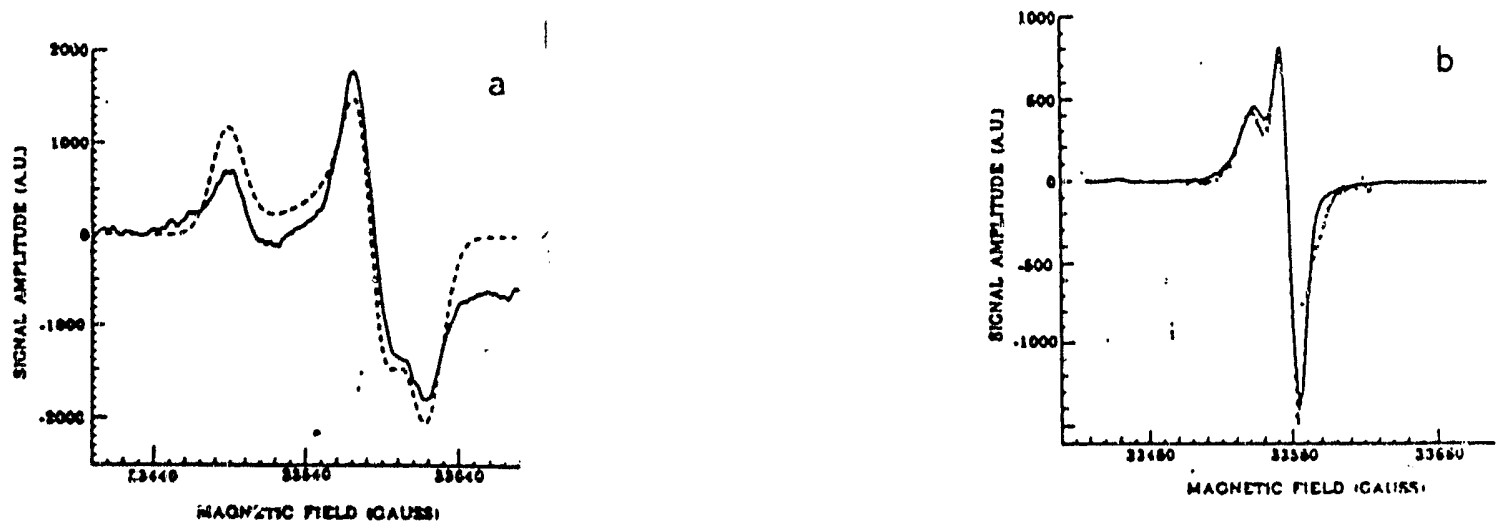

Figure 10. (a) W-band spectrum of dibenzothıophene $(+)($ ). ineorelicai simuiation (---). (iv) viirinite and composite spectrum (perylene $(+)$ and dibenzothiophene $(+)(\cdots)$. 
Because conjugated aromatics are the predominant structural types for organic sulfur in this coal. and because unpaired electrons are expected to be most abundant (and stable) in such chemical environments, it seems likely that EPR spectra from coal will strongly reflect the delccalization of electrons. This implies that a very small number of sulfur (or oxygen) atoms can exert a large effect on spectral lineshapes, making this technique uniquely sensitive to heteroatoms with targer SO coupling constants ( $\lambda$ ). Since the effect of a sulfur atom on $g$-values decreases as an electron is cielocalized over a larger and larger number of carbon atoms (and the $S / C$ ratio falls), the precise linesinapes trom sulfur in coal will depend on the size of the aromatic structures, as well as on the type of bonding. This fact represents another opportunity for the nondestructive analysis of high-sulfur coals by W-band EPR, since it ma;' be possible to model the spectra in order to get more detailed chemic al information about the forms of organic sulfur that are present. To illustrate this concept, Figure $1 \mathrm{C}(\mathrm{b})$ shows a W-band spectrum of vitrinite. Superimposed on it is a composite spectrum constructed by adding spectra from perylene $(+)$ and dibenzothiophene $(+)$. While the agreement between data and construct is not perfect, a comparison does suggest that molecular forms like perylene and dibenzothiophene are contributing to the experimental spectrum. In the future, we hope to use - pectral addition methods in a more quantitalive way to gain a better understanding of the molecular structure of the organic components of coal, including organic sulfur.

2) X-band ENDOR of model organic sulfur compounds

The analysis of coal macerals has posed a problem with resolution when using EPR spectroscopy. Coal samples examined at the traditional $X$-band microwave excitation frequency typically exhibit a single, nearly symmetric EPR abrorption line that is too fealureless to analyze uniquely. At $\mathrm{W}$. Jand $(95 \mathrm{GHz})$ however, anisotropic interactions in the g-matrix become apparent. The first $W$-band EPR experiment on coal unambiguously revealed the axial character of the system. The anisotropy of the g-matrix increases the chance of arriving at a unique theoretical description of the coal system.

In order to properly simulate the spectra obtained from coal-derived samples it becomes necessary start from fundamental building blocks of information. Sulfur containing systems inside coal are of special interest because of the concern over high sulfur content. Information about simpler sulfurcontaining molecules is needed 10 use in assembling the simulated spectra for coal samples. Thiophenes become the focus of this research eftort because most or yanic sulfur inside coal is in the form of thiophenes.

It was necessary to develop a consistent method to analyze the spectra obtained from the model systems with the emphasis on the anisotropic effects upon the EPR spectrum caused by sulfur. Thianthrene, a sulfur containing heterocyclic compound was a good model to begin with because it displays observable anisotropic features attributable $10 \mathrm{~g}$-matrix anisotropy in its X-band EPR signal when it is adsorbed onto the silica-alumina surface. Through the analysis of thianthrene's complex EPR and ENDOR spectra an analytical method of simulating disordered solid systems was developed. Since the anisotropic interactions in thianthrene are very complex, the method is applicable to highly disordered systems iri general and systems with less anisotropy. The focus of fulure experiments will be to apply this develnped method to thiophenes. We plan to characterize several thiophene class compounds that resemble the possible configurations of sulfur in coal.

Improvements must still be made on sample preparation techniques in order to obtain reproducible and reliable data from the analysis of the model systems. New surface treatment methods are currently being attempted to increase the EPR and ENDOR signals.

The modei sysiem thai wurkeu best fú the thianthirene oxporiment was produced by firs! calcining 
Houdry-M46 brand silica-alumina under vacuum and introducing the thiarithrene in the gas phase. The samples were prepared in quartz X-band EPR sample. EPR was performed on a Varian 12" Century Series EPR spectrometer with EISDOR capabilities. ENDOR spectra were taken at incremented magnetic field positions within the field range covered by the EPR signal.

The g-matrix was obtained by fitting the experimental EPR spectrum with a computer generated EPR spectrum. The parameters were optimized by using a auto-iterative routine that minimizes a least squares response function. The hyperfine matrices were obtained by optimizing the fit between the experimental ENDOR spectra and computer generated ENDOR spectra at various field positions.

Figures 11 and $12(\mathrm{a}-\mathrm{c})$ show results. Figure 11 compares the experimental EPR spectra and the best fit calculated by the method of Belford (Ref.[1]). The parameters are $g[1]=2.00146, g[2]=$ $2.00744, \mathrm{~g}[3]=2.01261$. Figure 12 compares experimental to calculated ENDOR spectra at the three field positions. There is a clear shift in hyperfine frequencies and intensities observed between the extreme field values. The distribution of molecular orientations relative to applied magnetic field coniributing to the ENDOR spectrum varies with field. That is, there is some angle selection at $X$-band although there is considerable overlap between the 3 turning points of the EPR spectrum. The best fit of the ENDOR spectra at all three field values required the assigning of the diagonal matrix g-values to be $g[x]=2.00744, g[y]=2.01261, g[z]=2.00146$.

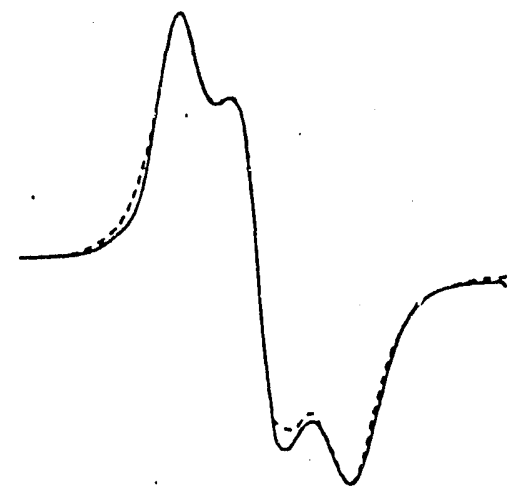

Figure 11. EPR spectrum of thianthrene (+). Experimental (____ $)$; theoretical (-..).

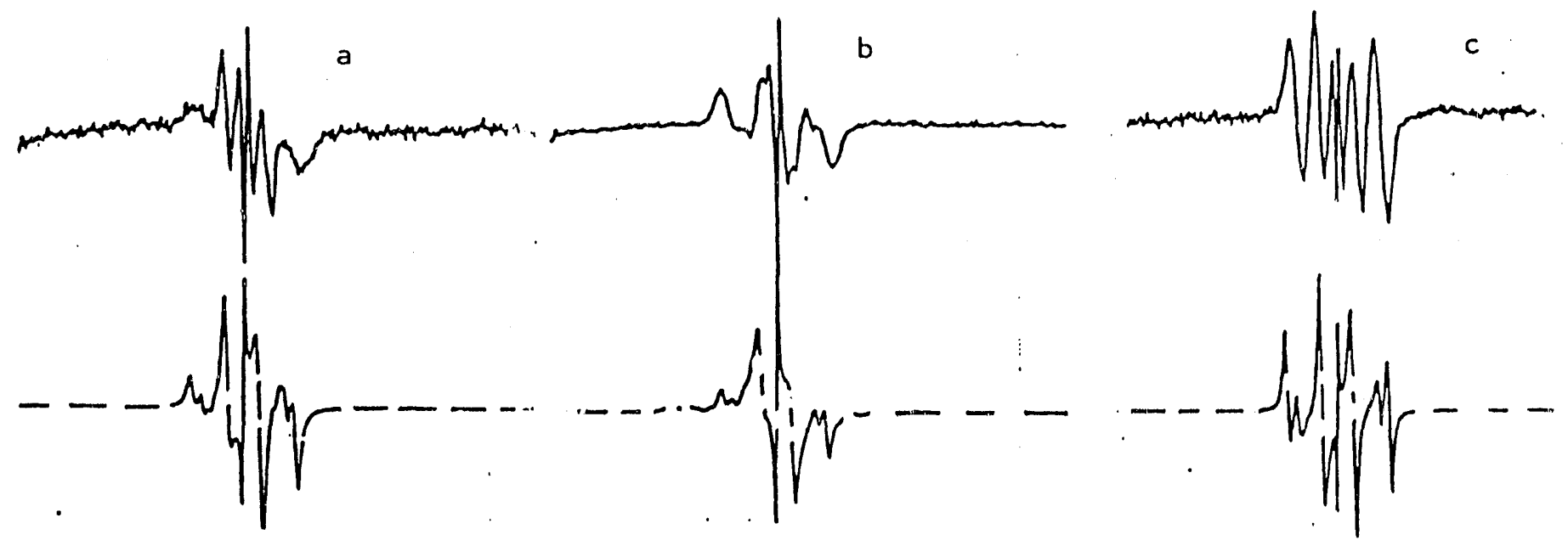

Figure 12. "Orientation selection" in proton ENDOR of thianthrene at three field positions. Experimental (__, theoretica! (-..). 
One conclusion that can be drawn is the assignment of the diagonal g-matrix elements $g[x], g[y], g[z]$ to the quantitative values calculated for the system and the consequent assignment of the directions of the $g$-values relative to the molecular geometry of the bound thianthrene. Figure 13 shows the present assignment for the directions of the numerical g-values. We must improve our calculation and continue refining the ENDOR parameters in order to be certain of this conclusion and to draw new conclusions about the model system.

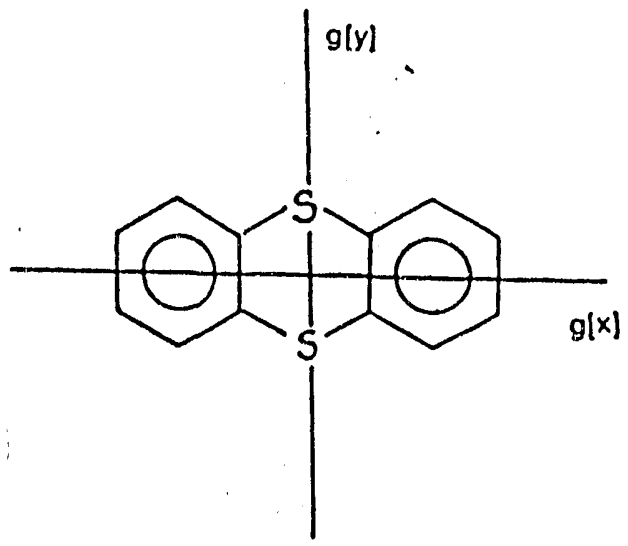

Figure 13. Assignment of directions of the canonical g-values in thianthrene.

The analysis described must be completed for thiophene compounds since thiophenes are bitter models for sulfur centers in coal. The preparation of thiophene models have been slightly more ciíificult in the past but have improved recently and better spectral signal to noise has been obtained. The computer algorithm used could also be improved by adding higher order correction terms to the spin Hamiltonian.

\section{3) Pulsed S-band EPR and ESE}

If ENDOR spectroscopy is the method of choice to resolve strong hyperfine interactions, ESE is uniquely sensitive to weak intramolecular interactions. In the first published account of ESE measurements in coal, Kevan et. al. studied matrix proton interactions, calculating a mean distance between unpaired electrons and protons in an SRC of $r=0.5 \pm 0.03 \mathrm{~nm}$, and an apparent interaction shell of $n=16 \pm 2$ protons [13]. Das et. al. found a value of $r=0.36 \pm 0.04 \mathrm{~nm}$ (n not reported) from a similar analysis of two-pulse ESE experiments performed on a low-sulfur Pittsburgh bituminous coal [22]. The matrix interaction, it will be remembered, is the essentially electron-nuclear dipole-dipole coupling between non-bonded atoms, and thus reflects the density of nuclei in the immediate neighborhood of the unpaired electron. The model used for these first analyses was a spherical shell, depicted in Figure 14. Such information, when correlated with specific maceral types, should provide us with a much more precise measurement of the atomic structure of coal, and may be diagnostic of structural changes brought about by various coal beneficiation technoloujies.

We have performed experiments on powdered Illinois $\# 6$ whole coal using a three-pulse ESE sequence, and have analyzed the matrix ESEEM by the spherical shell model. Figure 15 shows our data and best-fit simulation using $r=0.75 \pm 0.05 \mathrm{~nm}$ and $n=40$ protons [6]. Variations in $r$ and $\underline{n}$ among the three reported measurements may be due to differences in the structure of the samples studied; more likely sources of variations are the differences in experimental techniques (two-pulse, three-pulse), and different spectrometer characteristics (pulse power, cavity $Q$, etc.) used in the three sludies. We 
currently are making a detailed study of these effects using whole coals and coal model systems in an effort to better understand the role of experimental factors in the results obtained. We also are studying the utility of more realistic model geometries (eg, layered structure) and the sensitivity of ESEEM simulations to the choice of geometry.

Figure 14. The spherical shell model for analysis of matrix ESEEM.

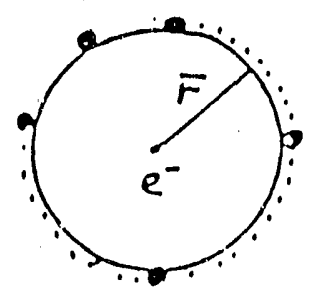

Figure 15. X-band ESEEM data, experimental ( ……......) and theoretical (-...) for an lllinois \#6 whole coal [6].

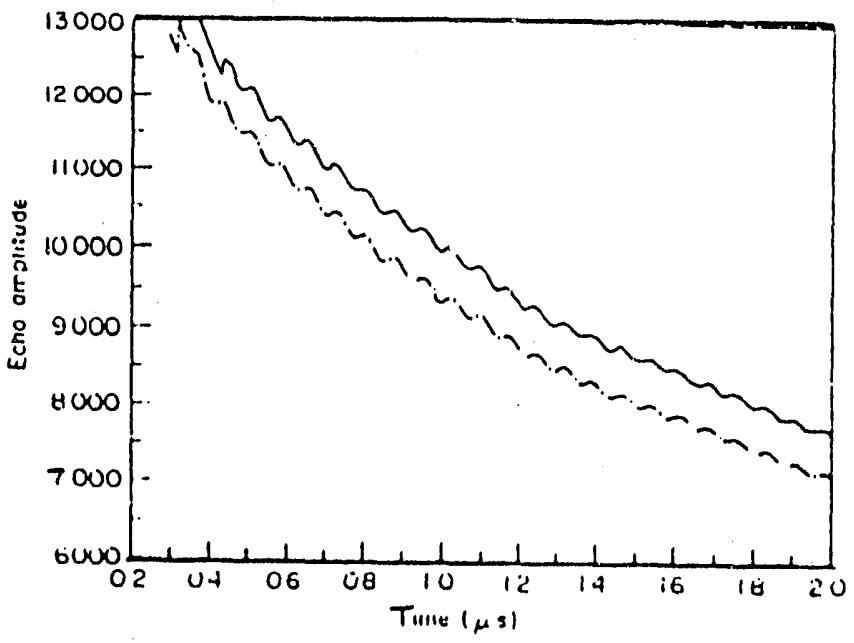

In 1984, we first reported the observation of matrix ESEEM from ${ }^{13} \mathrm{C}$ nuclei in natural abundance in coal [23]. Since that time, we have worked to understand the experimental and theoretical characteristics of this important effect, and we have recently developed criteria to optimize experimental sensitivity [24]. Because, to zeroth order, ESE sensitivity does not depend on the nuclear gyromagnetic ratio, this spectroscopy is much better suited for the observation of "low gamma" elements such as carbon and sulfur, which present severe problems for ENDOR and NMR. Data on ${ }^{3} \mathrm{C}$ ESEEM thus far suggests that in an Illinois \#6, $r=0.31 \pm 0.01 \mathrm{~nm}$, and $n=2$. Clearly, the opportunity to map the carbon skeleton of coal with this technique represents a great opportunity; it also is clear that much more work is needed to fully understand and correctly interprel such data.

\section{4) Computer-aided simulation}

Spectral parameters of EPR and related types of spectra are lypically oblained by simulating the experimental spectrum. Trial and error fitting of the spectral parameters can be time consuming and difficult especially if there are many adjustable spectral parameters and overlapping features. Often, as a result, the number of spectra that are feasible to simulate is severely limited, and it is difficult to arrive at a satisfactory fit. This is especially true of the ENDOR spectra of coal model systems. This has lead us to develop a method to assist in the fitting of simulation parameters.

We have designed a procedure to automate the fitting of spectral parameters in simulations. 
Each trial simulation is mathematically compared to the experimental spectrum and a new set of trial spectral parameters is chosen based on the results of all the previous simulations in the optimization. This process continues until a satisfactory simulation is obtained. The spectral parameters are chosen according to the simplex method of optimization. This method was chosen for use because its flexibility makes the program applicable to a variety of spectra. Thus, the fitting procedure can be used with any of our simulation programs to optimize the spectral parameters of EPR, ENDOR, and ESEEM spectra. Our goal is to use the optimization procedure routinely in the course of our data analysis both as a time saving device and as a way to insure that the most accurate spectral parameters result from our simulations.

The automation procedure which we have developed makes use of the simplex method of optimization. Simplex optimization can be used to vary any number of parameters to find the sel of values which gives the optimum response. The response is a function of the parameters which quantifies the results at each set of parameter values. The optimum is the maximum or minimum of the response function, depending on how it is defined. In this case the parameters are the variables in the simulations, the response is some measure of the match between the experimental spectrum and the simulation created with the set of parameter values that correspond to the vertex whose response is being measured, and the optimum would represent the values of the parameters leading to a simulation as neasly identical as possible to the experimental spectrum.

The simplex method employs an $n$-dimensional polyhedron, where $n$ is the number of variables being optimized. This polyhedron, the simplex, has $n+1$ vertices, each having $n$ coordinates which represent values of the variable parameters. For example, the simplex for an optimization of two variables $(x, y)$ is a triangle in which each of the three vertices has two coordinates which represent values of the two variables - i.e., $\left(x_{1}, y_{1}\right),\left(x_{2}, y_{2}\right),\left(x_{3}, y_{3}\right)$; the simplex for a three-variable optimization is a tetrahedron, and so on. After the initial simplex is determined, the response at each vertex is measured. The vertex with the worst response is replaced by its reflection through the hyperface of the remaining vertices. The response at the new vertex is measured and again the worst vertex is replaced. Repetition of this process causes the simplex to move over the response surface in the direction of the optimumras illustrated in Figure 16 for an optimization of two variables.

The modified simplex method was used in this project. It allows the worst vertex to be replaced not only by a reflected vertex but also by vertices that represent an expansion

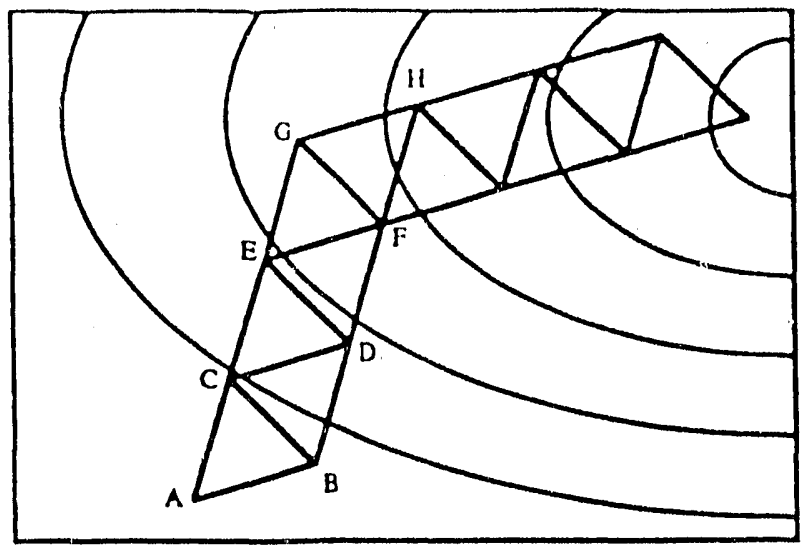

Figure 16: Movement of the simplex method on a two variable response surface. $A B C$ is the initial simplex. The vertex with the worst response, $A$, is replaced by its reflection, $D$. The simplex proceeds towards the optimum by replacing $B$ with $E, C$ with $F$, and $D$ with $G$. 
or contraction of the simplex. This allows the simplex to adjust its size in order to move more rapidly towards the optimum.

The response function used in the procedure can include any number of terms which quantify the goodness of fit between the experimental spectrum and the simulation. A least squares response provides a measure of the overall fit of the simulation. It is the sum over the data points of the squares of the differences between the experimental and simulated intensities. We have used this term as a response function both on its own and in combination with other terms which quantify how well the simulation reproduces the extrema of the experimental spectrum.

We have used the optimization procedure with a number of our simulation programs, including EPR powder spectra, ENDOR spectra with isotropic and snisotropic g-values, and ESEEM spectra. It has been shown to provide us with accurate spectral parimeters in a fraction of time previously needed for simulation. We are now able to more routinely simulate complicated spectra such as the ENDOR spectra that we obtain on polycyclic and polynuclear aromatic cations, our model systems for coal.

One of the systems we have studied using the optimizaticn procedure is the ENDOR spectra of polycyclic aromatic cations adsorbed on the surface of activated silica-alumina. In the case of coronene cations the hyperfine splittings could be easily estimated from the experimental spectrum. Reasonable bounds in a $2 \mathrm{MHz}$ range were assigned to each of the anisotropic components and the optimization was begun. Hyperfine values obtained from the optimization are 1.212, 2.598, and 6.354 $\mathrm{MHz}$. Figure 17 shows that the optimized simulation matches the experimental one well in overall appearance with only small discrepancies in the intensifies. We feel that the intensities are as well matched as possible given the level of theory in the present simulation program. Although this spectrum would not have been a particularly difficult spectrum to simulate, the optimization procedure worked well and saved time over a by-hand fit of the spectral parameters. It was also a very useful developmental tool for testing the procedure on an elementary case.

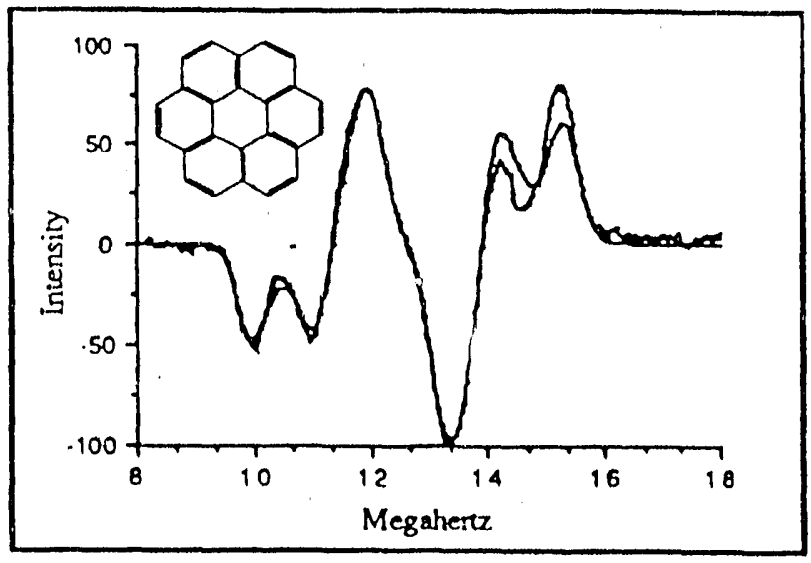

Figure 17: The ENDOR spectrum of coronene cations on silica-alumina and its simplex-generated simulation.

ENDOR spectra of the perylene crition include the effects of three sets of equivalent protons, each of which is anisotropic. The largest and smallest splittings are, like those of the coronene radical ion, easily approximated; however, overlap of the other teatures makes good estimation of the remaining hyperfine splitings nearly impossible. The hyperfine components are given bounds in a $2 \mathrm{MHz}$ range 
if they can be estimated and $4 \mathrm{MHz}$ if they cannol. The simulation obtained from an optimization of these values, shown in Figure 18, resembles the experimental spectrum quite well; the notable spectral features are reproduced with the only minor discrepancies in their relative intensities. Here a direct comparison with the time and effort necessary for a manual fit is possible because we had spent a considerable amount of time attempting to simulate this spectrum before the optimization procedure had been developed. The simplex procedure produces a slightly better simulation in a small fraction of the time and effort. This example is more representative of the types of difficult spectra which we hope to be able to simulate routinely with the automated procedure.

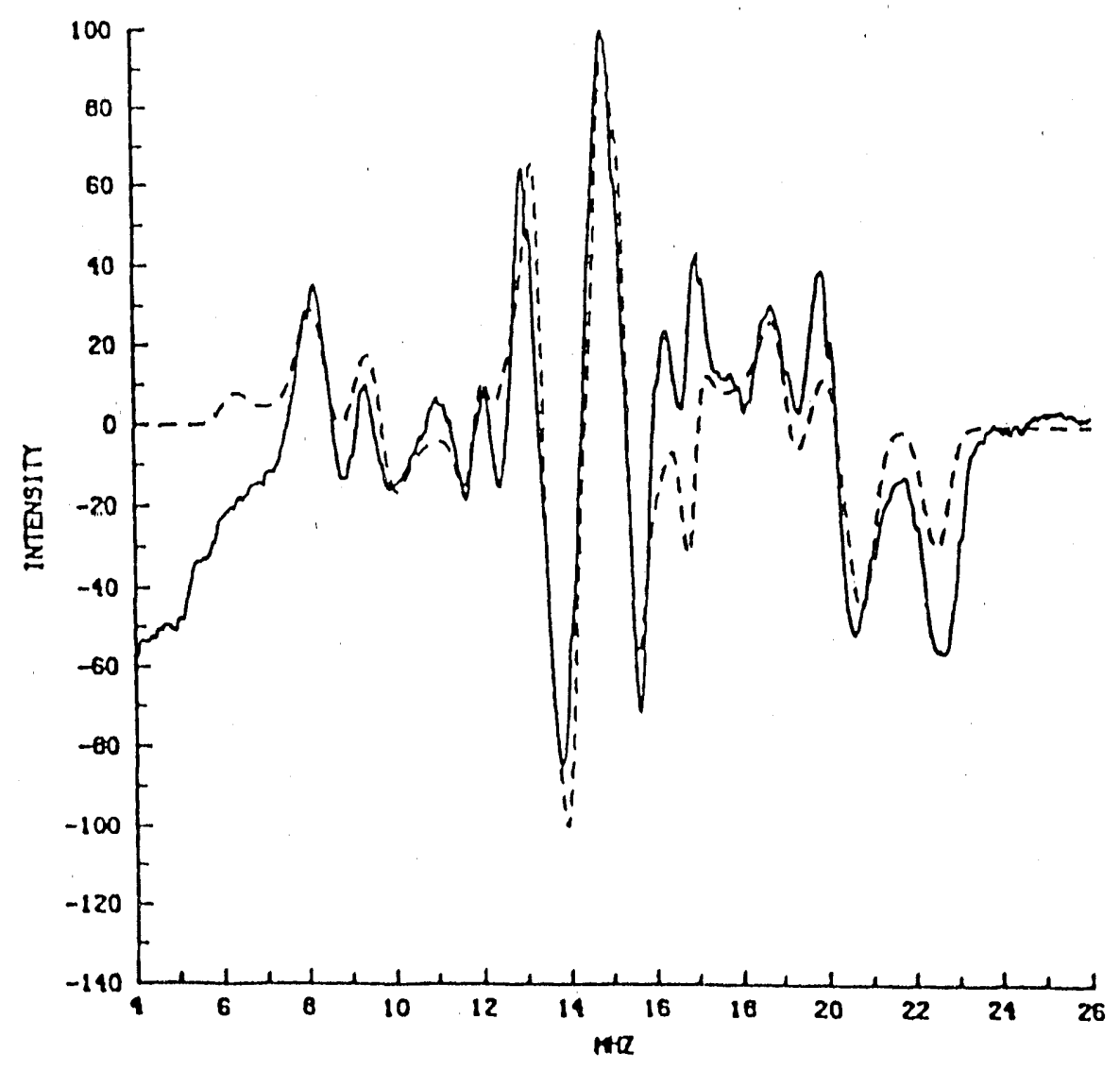

Figure 18: A comparison of the ENDOR spectrum of bound perylene and a Simplex-generated simulation.

5) MRI imaging of coal

The first proton images of coal have been made through the collaboration of Dr. Paul Lauterbur and the Biomedical Magnetic Resonance Laboratory (BMRL). A sample of lllinois \#6 was soaked overnight in DMSO (dimethyl sulfoxide). The solvent was chosen both for its properties as a good swelling solvent, and because it has six magnetically equivalent protons, resulting in a single proton NMR line (at low resolution). The sample was placed in a specially constructed microscopic NMR imaging probe, and a full three dimensional data set was accumulated. Preliminary results from this data suggest several pertinent tacts:

1) Good quality images can be produced of a solvent that has penetrated a normal coal sample. 
2) The images clearly show that there are regions in the coal that are not infiltrated by this solvent. Such regions comprise about $30 \%$ of the entire coal volume.

3) Resolution of better that 10 microns is already attainable by this method.

4) Regions where the solvent failed to penetrate have dimensions on the order of $20-80$ microns on a side.

Ve will make a full report on this new data, including images from these preliminary experiments, in the first quarterly report of the second year of this grant.

\section{CONCLUSIONS AND RECOMMENDATIONS}

1. W-band (96 GHz/3.4 T.) EPR resolves several peaks in an Illinois \#6 coal and separated macerals. Spectra from model compounds, together with theoretical simulations, suggest that these peaks represent contributions from chemically different species. One of the spectral components arises from a purely hydrocarbon species, which almost cerlainly has a conjugated aromatic structure. A second prominent peak can be accounted for very well by assuming a t'iophenic species. Vitrinite and sporinite macerals separated from the whole coal by density gradient centrifugation give different W-band EPR spectra thiat can be understood on the basis of differing organic sullur concentrations. The VHF EPR approach seems likely to allow the developinent of a reliable, non-destructive method to analyze for organic sulfur

In the coming year, we plan to continue improving the W-band spectrometer. In collaboration with Profs. Jack Crelling and Ed Hippo at Southern Illinois University, we will continue to study whole coals and separated macerals, acquiring more data to establish the analytical reliability of our spectral analysis to delermine organic sulfur concentration in coal. We also will sludy coals from the Argonne Premium Coal Sample Program, in order to take advantage of the wealth of analytical data that is available for these coals. Power saturation studies should be carried out on selected samples in order to explore the usefulness of power and phase techniques in improving still further the resolution of different chemical components of whole coal. In the future, EPR at still higher frequencies should be explored as an analytical method to characterize coal. Also, the possibility of adding ENDOR capabilities to the $W$-band instrument should be investigated.

2. ENDOR studies on thianthrene and dibenzothiophene confirm that g-anisotropy in these systems brought about by spin-orbit coupling with sulfur can be a mechanism for "orientation selection" at $X$ band. Theoretical simulations confirm the adequacy of our molecular model for this process, allowing us to predict the nature of spectra exhibiting this interaction.

The great opportunity to exploit "orientation selection" in organic sulfur-containing systems as an analytical tool must wait for higher field ENDOR instrumentation. W-band ENDOR should demonstrate a really strong effect. It is recommended that work begin on developing a W-band ENDOR capability.

3. S-band pulsed EPR and ESE continue to develop experimentally and theoretically as useful and powerful tools for determining the atomic structure of coal. Parallel studies at S-and X-band in our laboratory and in laboratories at the Argonne National Laboratory, Albert Einstein College of Medicine, Cornell University, and at the ETH. Zürich confirm that our experimental and theoretical methods are accurate in determining positions, numbers, and orientations of atoms in coal. Multi-frequency ESE measurements continue to be vital for accurate interpretation of the data. 
Work must continue on developing better timing control circuits for our S-band pulsed EPR/ESE spectrometer. New software to allow the programming of more complex experiments also should be completed in the near future. We then plan to make a detailed study of separated macerals and coals that have been subjected to desulfurization, in order to learn more about the structural effects of such processes.

4. Simplex optimization techniques allow a significant reduction in the time it takes to fit experimental spectra with a theoretical calculation. They will be added to all of our modeling software

5. MRI of coal provides information not available by any other technique. Our preliminary data on solvent penetration suggests that both NMR and EPR imaging wili be useful. In the coming year, we plan to continue to develop this method for coal. The preliminary solvent data suggest that MRI may allow determination of the extent and rate of solvent penetration in coals.

\section{REFERENCES}

1. W. H. Wiser and R. H. Wolk, ACS Fuel Div. Prepts., 20 (2), 122 (1975).

2. R. M. Davidson, Molecular Struclure of Coal, IEA Coal Research, London, 1980.

3. J. H. Shinn, FUEL, $\underline{63}, 1187$ (1984).

4. L. Lazarov and S. P. Marinov, Fuel Proc. Technol., 15, 411 (1987).

5. K. J. Hu"ttinger and A. W. Michenfelder, FUEL; 66, 1164 (1987).

6. J. Uebersfeld, A. Etienne, and J. Combrisson, Nature, Lond., 174, 615 (1954).

7. D. J. E. Ingram, J. G. Tapley, R. Jackson, R. L. Bond, and A. R. Murnagham, Nature, Lond., 174, 797 (1954)

8. H. L. Retcofsky, M. R. Hough, M. M. Maguire, and R. B. Clarkson, in Coal Structure, M. L. Gorbaty and K. Ouchi, eds., ACS Advances in Chemistry, 192, ACS, Washington, 1981, pp. 37 - 58.

9. R. B. Clarkson, R. L. Belford, K. S. Rothenberger, and H. C. Crookham, J. Catalysis, 106, 500 (1987).

10. R. B. Clarkson, R. L. Belford, J. B. Cornelius, P. A. Snetsinger, and M. K. Bowman, FUEL, 66, 925 (1987).

11. L. Kevan and R. N. Schwartz, Time Domain Electron Spin Resonance, John Wiley \& Sons, New York, 1979.

12. K. Ohno and T. Yokono, Carbon, 24, 517 (1986).

13. L. J. Berliner, H. Fujii, X. Wan, and S. J. Lukiewicz, Mag. Res. Med., 4, 380 (1987).

14. M. M. Maltempo, S. S. Eaton, and G. R. Eaton, J. Mag. Res., 2, 449 (1987).

15. W. Brian Lynch, Keith A. Earle, and Jack H. Freed, Rev. Sci. Instrum., 59 (1988) 1345.

16. A. A. Galkin, G. G. Grinev, V. I. Kurochkin, and E. D. Nemchenko, Delekloskopiya, 4 (1976) 121. 
17. R. J. Wagner and A. M. White, Solid State Commun., 32 (1979) 399.

18. R. T. Weber, J. A. J. M. Disselhorst, L. J. Prevo, J. Schmildt, and W. Th. Wenckebach, J. Mag. Res., 81 (1989) 129.

19. A. J. Stone, Mol. Phys., 7 (1964) 311.

20. R. B. Clarkson, R. L. Belford, K. S. Rothenbergor, and H. C. Crookham, J. Catalysis, 106 (1987) 500 .

21. A. Attar and F. Dupuis, ACS Div. Fuel Chem. Preprints, 24 (1979) 166.

22. U. Das, D. C. Doetschman, P. M. Jones, and M. Lee, FUEL, 6’., 285 (1983).

23. "The Interactions of Paramagnetic Centers with ${ }^{13} \mathrm{C},{ }^{1} \mathrm{H}$, and Adsorbed Oxygeis in Coal," $\mathrm{R}$. B. Clarkson and M. K. Bowman, 7th International EPR Symposium, Denver, 1984, paper no. 87.

24. P. A. Snetsinger, J. B. Cornelius, R. B. Clarkson, and Fi. L. Belford, J. Phys. Chem., 1987

\section{DISCLAIMER}

This report was prepared as an account of work sponsored by an agency of the United States Government. Neither the United States Government nor any agency thereof, nor any of their employees, makes any warranty, express or implied, or assumes any legal liability or responsibility for the accuracy, completeness, or usefulness of any information, apparatus, product, or process disclosed, or represents that its use would not infringe privately owned rights. Reference herein to any specific commercial product, process, or service by trade name, trademark, manufacturer, or otherwise does not necessarily constitute or imply its endorsement, recommendation, or favoring by the United States Government or any agency thereof. The views and opinions of authors expressed herein do not necessarily state or reflect those of the
United States Government or any agency thereof. 

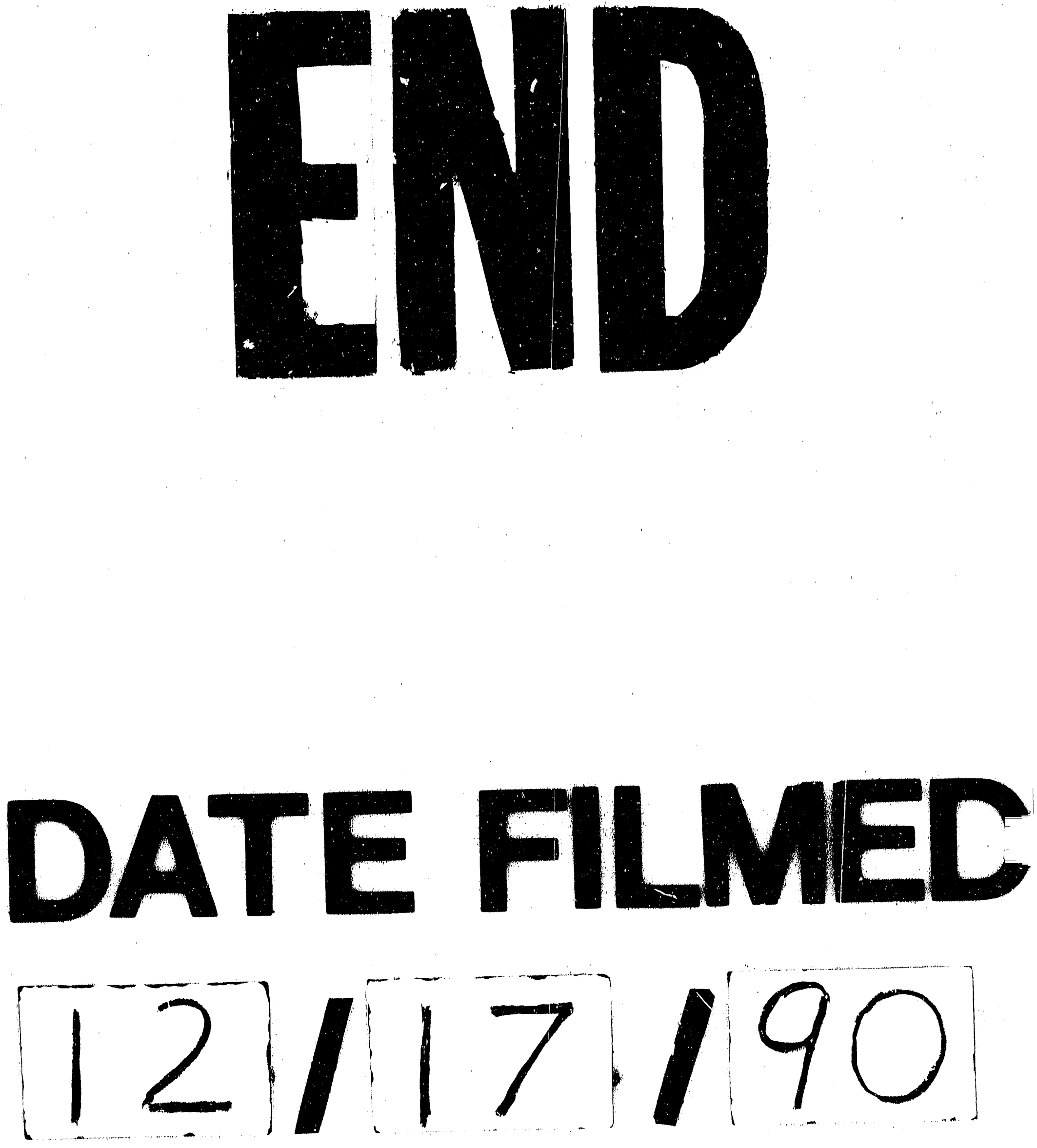
